\title{
PROGNOSTIC FACTORS
}

\author{
W R Miller, I O Ellis, J R C Sainsbury, J M Dixon
}

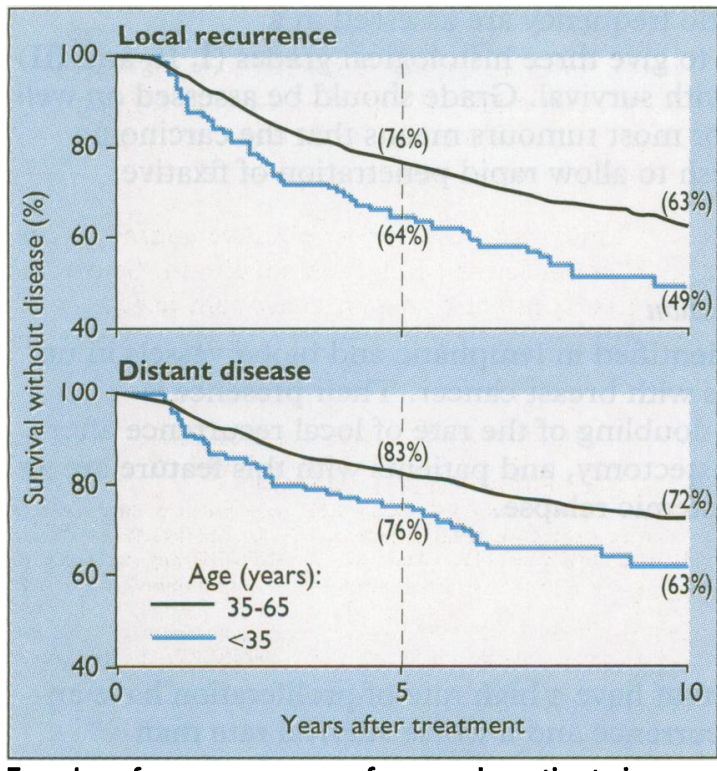

Freedom from recurrence of cancer in patients in relation to age when breast cancer first diagnosed. (Proportional hazards model showed age $<35$ to have relative risk of 1.6 for distant disease.)

\section{Chronological factors}

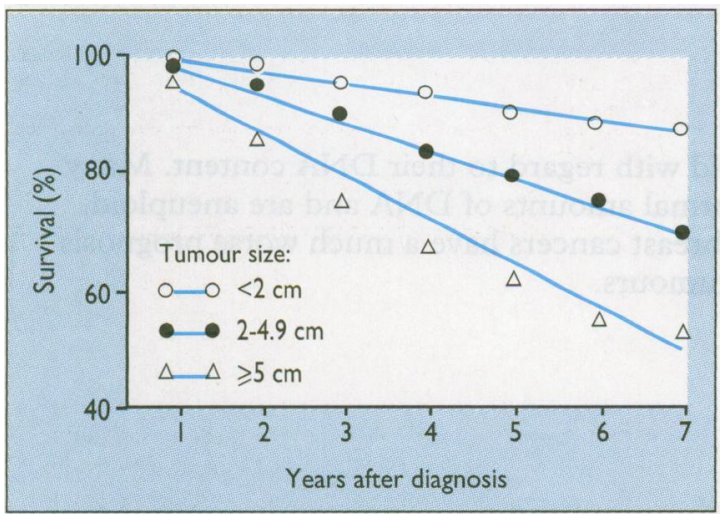

Survival in relation to size of breast cancer.
Prognostic factors are of value for three main reasons:

- To help select the appropriate treatment for individual patients

- To allow comparisons of treatments between groups of patients at similar risks of recurrence or death

- To improve our understanding of breast cancer, which may permit the development of new strategies or treatments

Prognostic factors can be broadly classified into two groups: chronological factors, which are indicators of how long the cancer has been present and relate to stage of disease at presentation, and biological factors, which relate to the intrinsic or potential behaviour of the tumour. However, recent evidence suggests that age at diagnosis may also be a risk factor: younger women (aged under 35 ) have a poorer prognosis than older patients with cancer of equivalent stage.

\section{Tumour size}

The pathological size of a tumour correlates directly with survival; patients with smaller tumours have a better survival rate than those with large tumours. Maximum pathological size should be assessed in fresh specimens, and the size should be subsequently confirmed or amended after histological examination.

\section{Status of axillary lymph nodes}

The single best prognostic factor is the presence or absence of axillary nodal metastases. There is a direct correlation between survival and the number of axillary lymph nodes involved.

\section{Metastases}

Patients in whom cancer has spread beyond the axillary or internal mammary nodes ( $M_{1}$ or stage IV disease) have a much worse survival rate than patients whose disease is apparently localised. There are differences in survival between patients depending on the site of the metastatic disease, with patients who have supraclavicular involvement as their only site of metastases having a much better survival rate than patients with metastases at other sites.

\section{Survival of patients with breast cancer according to involvement of axillary lymph nodes}

\section{All patients}

Negative axillary lymph nodes

Positive axillary lymph nodes:

1-3

$\geqslant 4$
Survival at 10 years

$45.9 \%$

$64.9 \%$

$24.9 \%$

$37.5 \%$

$13.4 \%$

\begin{tabular}{|c|c|}
\hline \multicolumn{2}{|c|}{$\begin{array}{l}\text { Survival of patients according to } \\
\text { stage of tumour }\end{array}$} \\
\hline Stage & Survival at 5 years \\
\hline & $84 \%$ \\
\hline II & $71 \%$ \\
\hline III & $48 \%$ \\
\hline IV & $18 \%$ \\
\hline
\end{tabular}




\section{Biological factors}

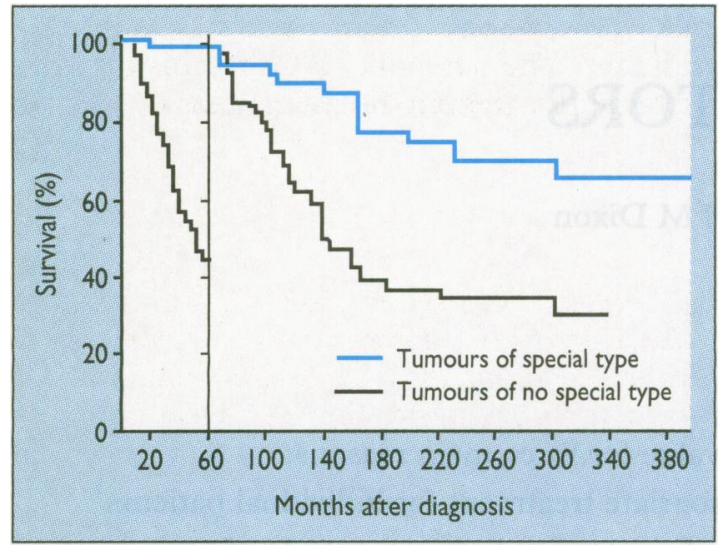

Short and long term survival in relation to histological type of breast cancer.

\section{Survival of patients according to histological grade of tumour}

\begin{tabular}{ll} 
Histological grade & Survival at 10 years \\
I & $85 \%$ \\
II & $60 \%$ \\
III & $40 \%$ \\
\hline
\end{tabular}

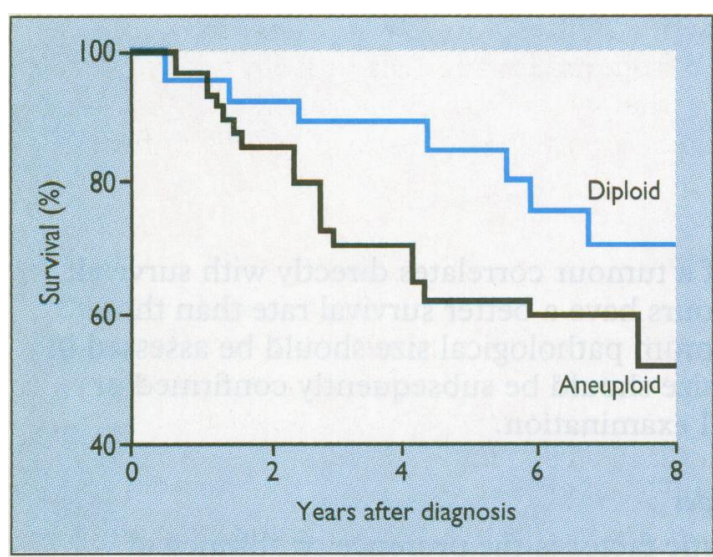

Survival in relation to DNA content of breast cancer.

\section{Biochemical measurements}

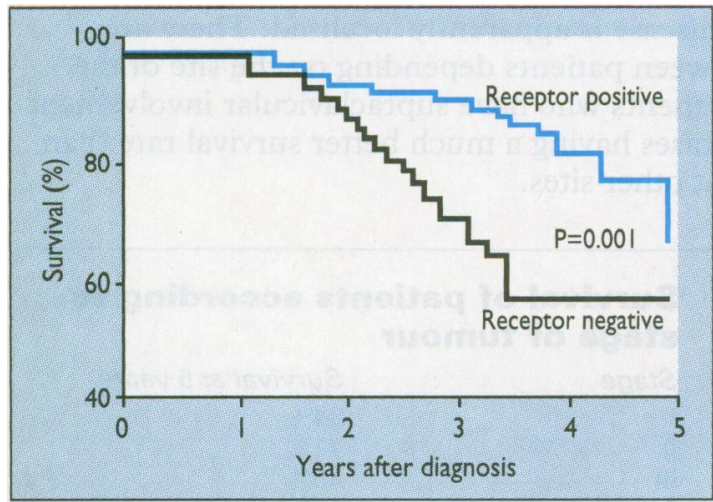

Survival in relation to concentration of oestrogen receptor in breast cancer.

\section{Histological type}

Many of the so called special types of invasive breast carcinoma (invasive tubular, cribriform, mucinous, papillary, and microinvasive) are associated with a much better prognosis than cancers of no special type. Histological type is one of the best predictors of long term survival.

\section{Histological grade}

The three characteristics of tubular formation, nuclear pleomorphism, and mitotic frequency are assessed in a semiquantitative manner to give three histological grades (I, II, and III) which correlate directly with survival. Grade should be assessed on well fixed specimens, which for most tumours means that the carcinoma should be sliced while fresh to allow rapid penetration of fixative.

\section{Lymphatic or vascular invasion}

Tumour cells can be identified in lymphatic and blood vessels in up to a quarter of all patients with breast cancer. Their presence is associated with at least a doubling of the rate of local recurrence after wide local excision or mastectomy, and patients with this feature are at high risk of short term systemic relapse.

\section{Markers of proliferation}

Patients with tumours that have a high rate of proliferation have an increased rate of local recurrence and a worse survival rate than patients whose tumours proliferate slowly. Several methods to measure proliferation have been reported, including measurement of the fraction of cells in the $S$ phase of the cell cycle; the use of monoclonal antibodies such as Ki67, KiS1, and MIB-1; and identification of proliferating cells by the use of tracers such as bromodeoxyuridine. Measurement of proliferation alone does not give complete information about a tumour because in each tumour there is a balance between proliferation and cell loss and because prognosis depends not only on the rate of proliferation but on the metastatic potential of a breast cancer.

\section{DNA content of a tumour}

Normal cells are diploid with regard to their DNA content. Many breast cancers have abnormal amounts of DNA and are aneuploid. Patients with aneuploid breast cancers have a much worse prognosis than those with diploid tumours.

\section{Hormone and growth factor receptors}

The presence of oestrogen receptors in a breast cancer predicts response to hormonal manipulation; this appears to be of some value in predicting early outcome after treatment but is of limited value in predicting long term survival. Progesterone receptors can be identified in some breast cancers; their presence depends on an intact oestrogen receptor pathway, but it is not clear that they are of more value than oestrogen receptors in predicting prognosis or response to hormonal treatment. 


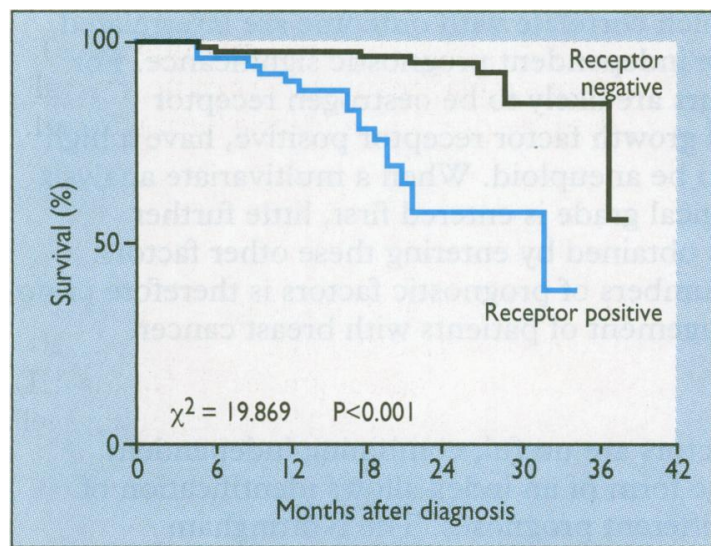

Survival in relation to concentration of epidermal growth factor receptor in breast cancer.

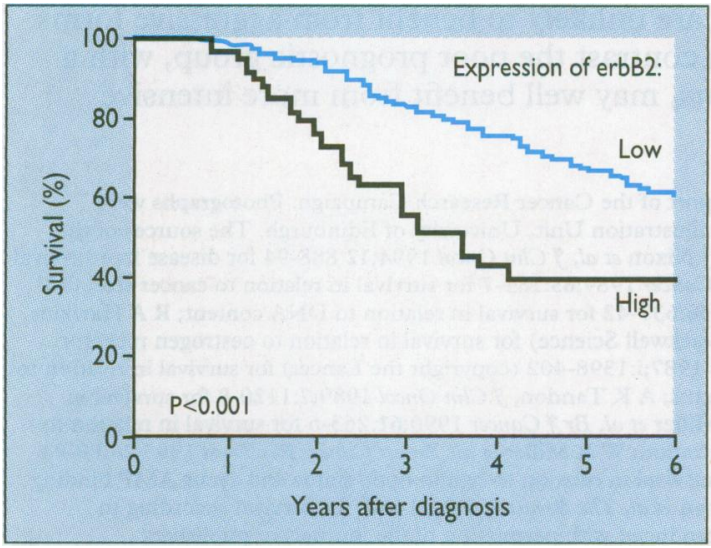

Survival of patients with breast cancer and involved axillary lymph nodes in relation to expression of oncogene erbB2.
The presence of epidermal growth factor receptors within the membrane of breast cancer cells is inversely correlated with the presence of oestrogen receptors and is associated with a diminished period free of relapse and reduced overall survival. Patients whose tumours are positive for epidermal growth factor receptors are unlikely to respond to hormonal treatment. The possibility of using this growth factor receptor pathway as a target for treating breast cancers is currently being investigated.

\section{Oncogenes}

The proto-oncogene erbB2 is overexpressed in 15-30\% of invasive cancers and in up to $80 \%$ of non-invasive cancers, and its product is homologous with the epidermal growth factor receptor. Patients with lymph node involvement whose tumours express erbB2 have a particularly poor prognosis, but erbB2 seems to be of less value in delineating the prognosis of patients who are lymph node negative. Tumours which express erbB2 are more likely to be resistant to both chemotherapy and hormonal treatment.

\section{Tumour suppressor genes}

p53 Is a product of a gene found on the short arm of chromosome 17. Its abnormal expression is the most common genetic lesion detected in breast cancers, and one group of patients who have a greatly increased risk of breast, ovarian, and bowel cancer (those with Li-Fraumeni syndrome) have abnormal p53 expression. The product of the gene seems to be a transcription factor responsible for checking the fidelity of cell replication.

\section{Proteases}

Cathepsin D is a protease capable of degrading basement membrane. It is not clear whether the cathepsin D found in breast cancers is primarily derived from malignant cells or from macrophages. Early studies suggested that the presence of cathepsin D in a tumour correlated with prognosis, but recent studies have failed to confirm this.

\section{Second messenger systems}

Cyclic AMP binding proteins are the regulatory subunits of a major second messenger system - protein kinase A. High concentrations of cyclic AMP binding proteins are present in 10-15\% of breast cancers, and patients with these cancers have a very poor survival rate. The concentration of cyclic AMP binding protein can be used to identify a subgroup of patients who do not have axillary node involvement but yet have a very poor outlook.

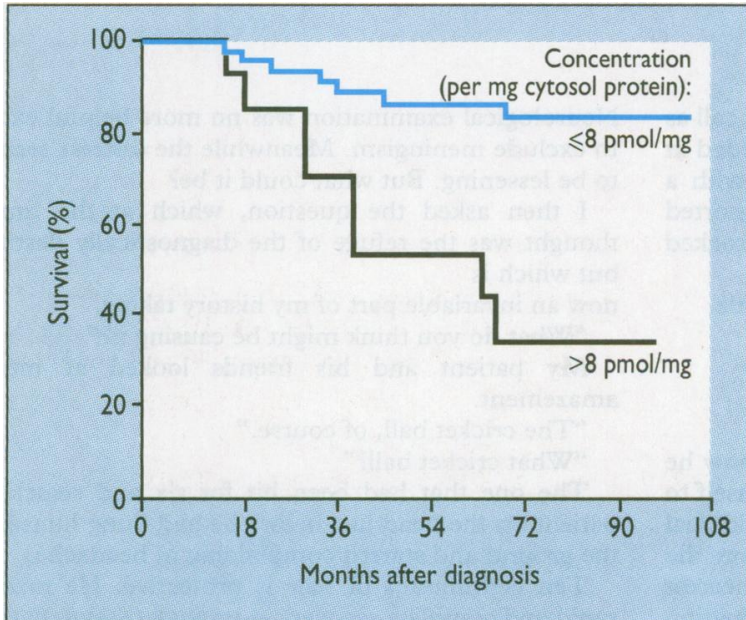

Survival in relation to concentration of cyclic AMP binding proteins in breast cancer.

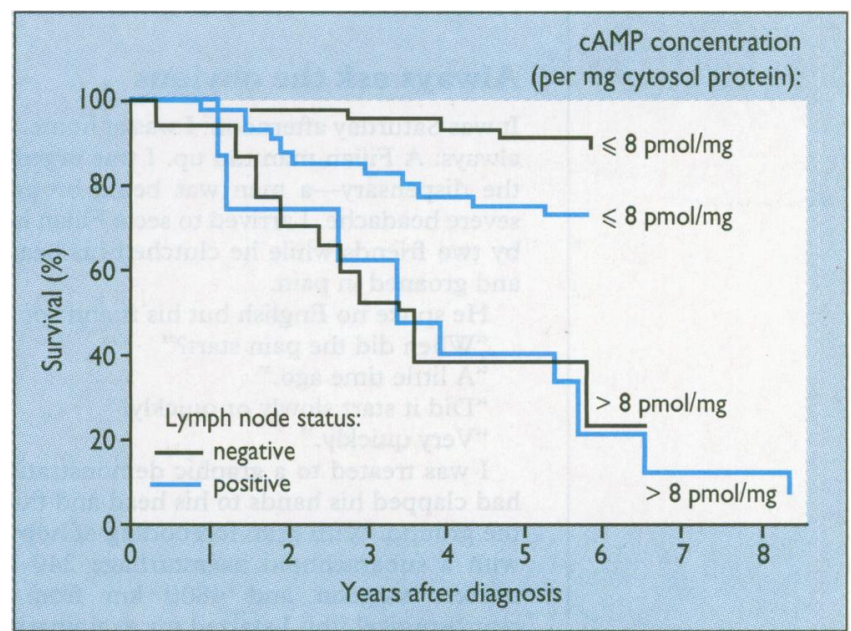

Survival in relation to axillary lymph node status and concentration of cyclic AMP binding proteins in breast cancer. 


\section{Nottingham prognostic index=}

$(0.2 \times$ size $)+$ lymph node stage+grade

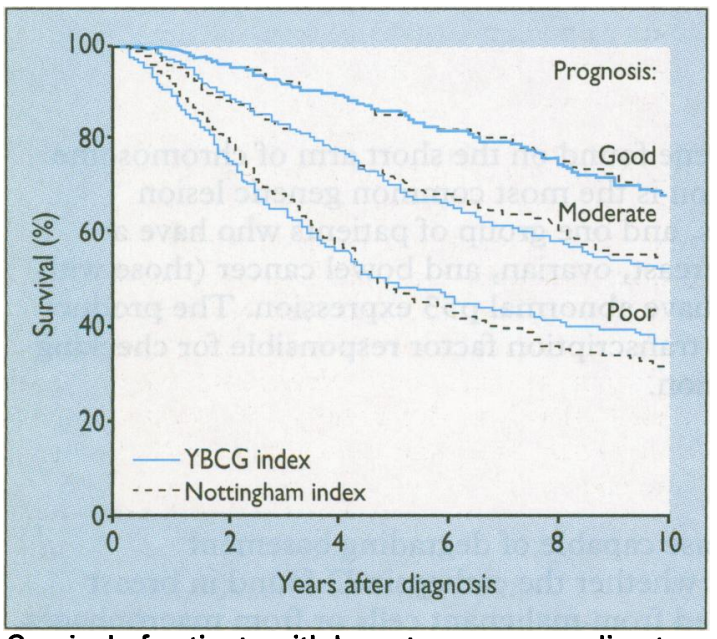

Survival of patients with breast cancer according to Nottingham and Yorkshire Breast Cancer Group prognostic indices.

\section{Interrelated factors}

Many of the factors which correlate with outcome are interrelated and do not therefore have independent prognostic significance. For example, grade III tumours are likely to be oestrogen receptor negative, to be epidermal growth factor receptor positive, have a high proliferative index, and to be aneuploid. When a multivariate analysis is performed and histological grade is entered first, little further prognostic information is obtained by entering these other factors. Measurements of large numbers of prognostic factors is therefore of no value in the routine management of patients with breast cancer.

\section{Prognostic indices}

Although individual factors are useful, combining independent prognostic variables in the form of an index allows identification of groups of patients with different prognoses. The Nottingham prognostic index is the most widely used index and incorporates three prognostic factors: tumour size, node status, and histological grade.

With the Nottingham index the lymph node stage is 1 if no nodes are involved, 2 if one to three nodes are involved, and 3 if four or more nodes are involved. The Yorkshire Breast Cancer Group categorised lymph node stage as 1 if no nodes were involved or 3 for any axillary node involvement. The Yorkshire group also used different codes for tumour grade: code 1 for grade I and code 2 for grades II and III. Both indices identify three prognostic groups. The good prognostic group has a survival similar to that of age matched controls without breast cancer, and such women are unlikely to benefit from aggressive forms of adjuvant treatment. In contrast the poor prognostic group, with a $13 \%$ survival after 15 years, may well benefit from more intensive systemic treatment.

J M Dixon acknowledges the support of the Cancer Research Campaign. Photographs were prepared by Mr D Dirom, Medical Illustration Unit, University of Edinburgh. The sources of the data presented in the graphs are: A J Nixon et al, $\mathcal{F}$ Clin Oncol 1994;12:888-94 for disease free survival in relation to age; C L Carter et al, Cancer 1989;63:181-7 for survival in relation to cancer size; O-P Kallioniemi et al, Br $\mathcal{f}$ Cancer 1987;56:637-42 for survival in relation to DNA content; R A Hawkins et al, Br F Surg 1987;74:1009-13 (Blackwell Science) for survival in relation to oestrogen receptor status; J R C Sainsbury et al, Lancet 1987;i:1398-402 (copyright the Lancet) for survival in relation to epidermal growth factor receptor status; A K Tandon, $\mathcal{F}$ Clin Oncol 1989;7:1120-8 for survival in relation to erbB2 expression; W R Miller et al, Br f Cancer 1990;61:263-6 for survival in relation to cyclic AMP binding protein concentration; W R Miller et al, Breast Cancer Res Treat 1993;26:89-94 (Kluwer Academic Publishers) for survival in relation to lymph node status and cyclic AMP binding protein concentration; and J M Brown et al, The Breast 1993;2:144-7 for survival according to prognostic indices. The data are reproduced with permission of the journals or publishers.

W R Miller is deputy director of ICRF Medical Oncology Unit, Western General Hospital, Edinburgh; I O Ellis is consultant histopathologist at Nottingham City Hospital; J R C Sainsbury is consultant surgeon at Huddersfield Royal Infirmary; and J $\mathrm{M}$ Dixon is senior lecturer in surgery at Edinburgh Royal Infirmary and honorary consultant surgeon at Western General Hospital, Edinburgh.

The ABC of Breast Diseases has been edited by J Michael Dixon.

\section{A PATIENT WHO CHANGED MY PRACTICE}

\section{Always ask the obvious}

It was Saturday afternoon. I was at home, but on call as always. A Fijian man ran up. I was urgently needed at the dispensary - a man was being brought in with a severe headache. I arrived to see a Fijian half supported by two friends while he clutched his head and rocked and groaned in pain.

He spoke no English but his friend spoke a little.

"When did the pain start?"

"A little time ago."

"Did it start slowly or quickly?"

"Very quickly."

I was treated to a graphic demonstration of how he had clapped his hands to his head and flung himself to the ground. With grim foreboding of how I would deal with a subarachnoid haemorrhage $240 \mathrm{~km}$ from the nearest surgeon and $4800 \mathrm{~km}$ from the nearest neurosurgical unit I started my examination. Palpation of the skull through $10 \mathrm{~cm}$ of hair was unhelpful.
Neurological examination was no more helpful except to exclude meningism. Meanwhile the distress seemed to be lessening. But what could it be?

I then asked the question, which at the time I thought was the refuge of the diagnostically destitute but which is

now an invariable part of my history taking.

"What do you think might be causing it?"

My patient and his friends looked at me in amazement.

"The cricket ball, of course."

"What cricket ball?"

The one that had been hit for six and struck my patient on the head just before he had flung himself to the ground and started complaining of headache.

Ten centimetres of hair is protective. He made a rapid and complete recovery.-HARVEY J MARRABLE is a psychiatrist in New South Wales 